\title{
Exploiting night-time averaged spectra from PFS/MEX shortwave channel. Part 2: near-surface CO retrievals
}

\author{
Sophie Bauduin ${ }^{\mathrm{a}}$, Marco Giuranna ${ }^{\mathrm{b}}$, Paulina Wolkenberg ${ }^{\mathrm{b}}$, Luca Nardic, ${ }^{\mathrm{b}}$, \\ Frank Daerden ${ }^{\mathrm{d}}$, Jimmy Bouche ${ }^{\mathrm{a}}$, Catherine Wespes ${ }^{\mathrm{a}}$, Gilles Lecomte ${ }^{\mathrm{a}}$, Ann \\ Carine Vandaele ${ }^{\mathrm{d}}$, Pierre Coheur ${ }^{\mathrm{a}}$ \\ ${ }^{a}$ Université libre de Bruxelles (ULB), Spectroscopy, Quantum Chemistry and \\ Atmospheric Remote Sensing (SQUARES), Brussels, Belgium \\ ${ }^{b}$ National Institute of Astrophysics INAF-IAPS, Rome, Italy \\ ${ }^{c}$ University of Rome "La Sapienza", Rome, Italy. \\ ${ }^{d}$ Royal Belgian Institute for Space Aeronomy, Brussels, Belgium
}

\section{Abstract}

Because of its important role in the Martian carbon cycle, carbon monoxide $(\mathrm{CO})$ has been the subject of many measurements from ground and from space. Daytime measurements have been mostly exploited to measure the CO abundance because of their good signal-to-noise ratio, but night-time observations have not been documented yet. We demonstrate here the possibility of using PFS (Planetary Fourier Spectrometer) night-time observations to measure the Martian CO abundance. More particularly in this paper, we show that measuring CO during night with PFS can be achieved by averaging a large number of spectra to reach sufficient signal-to-noise. Furthermore, we show that the number of averaged spectra is not the only driving parameter for the detection of CO. High surface temperatures and high thermal contrast (negative in our case) are the two other conditions required for the measurement of the night-time CO abundance. Because of this, the retrievals are especially successful in the Southern Hemisphere during spring and sum- 
mer when and where these two conditions are met. For night-time spectra with a positive detection, $\mathrm{CO}$ vertical profiles are successfully retrieved using the Optimal Estimation method and are characterized in terms of vertical sensitivity. Successful retrievals imply the use of accurate temperature profiles, and in particular an appropriate representation of the thermal inversion in the lower atmosphere. The temperature was obtained by using the relevant information from the $\mathrm{CO}_{2} \nu_{3}$ band (Bauduin et al., 2020, this issue). A complete error budget of the retrieved $\mathrm{CO}$ profiles is also performed and includes different sources of uncertainty. Although the retrieved profiles are not resolved vertically, we show in particular that night-time PFS observations carry information mostly on the $\mathrm{CO}$ abundance for the $0-10 \mathrm{~km}$ altitude region, and thus provide a stronger constraint on the near-surface $\mathrm{CO}$ abundance compared to daytime observations.

Keywords: Mars atmosphere, carbon monoxide, night measurements, retrieval, PFS

\section{Introduction}

2 Carbon monoxide $(\mathrm{CO})$ plays a major role in the carbon cycle of the Mars' 3 atmosphere, notably due to its involvement in the so-called stability prob4 lem of carbon dioxide $\left(\mathrm{CO}_{2}\right)$ (McElroy and Donahue, 1972; Parkinson and 5 Hunten, 1972; Atreya and Gu, 1994, 1995). Since its first detection in 1969 6 (Kaplan et al., 1969), CO has been extensively measured from ground (e.g. 7 Clancy et al., 1983; Krasnopolsky, 2003, 2015) and from different space-borne 8 instruments, such as OMEGA (Observatoire pour la Minéralogie, l'Eau, les 9 Glaces et l'Activité) (Encrenaz et al., 2006), CRISM (Compact Reconnais- 
sance Imaging Spectrometer for Mars) (e.g. Smith et al., 2018) and PFS (Planetary Fourier Spectrometer) (e.g. Billebaud et al., 2009; Sindoni et al., 2011; Bouche et al., 2019). These ground and space observations have revealed a more complete picture of the $\mathrm{CO}$ climatology. Indeed, $\mathrm{CO}$ is not uniformly distributed in the Martian atmosphere, but shows strong latitudinal and seasonal gradients related to the condensation and sublimation of $\mathrm{CO}_{2}$ at the poles. Although these spatial and time variations are today relatively well measured and understood, the vertical dimension is still lacking in this climatology. Different global circulation models are able to simulate the vertical distribution of $\mathrm{CO}$ and its seasonal/spatial variations (e.g. Forget et al., 1999, 2008; Daerden et al., 2019; Holmes et al., 2019), but measurements of the CO vertical profiles from remote sounders remain rare. Retrievals of highly resolved CO profiles from solar occultation observations of NOMAD (Nadir and Occultation for Mars Discovery) (Vandaele et al., 2018) and ACS (Atmospheric Chemistry Suite) (Korablev et al., 2018) instruments on board the ExoMars Trace Gas Orbiter (TGO) have started (Erwin et al., 2019; Olsen et al., 2019) and should provide in a near-future a better understanding of the vertical variability of CO in Mars' atmosphere. Nevertheless, solar occultation measurements are generally limited to the sounding of the atmosphere above 10-20 km and thus cannot provide CO measurements for the near-surface atmosphere. Recently, Bouche et al. (2019) have investigated the possibility of retrieving $\mathrm{CO}$ profiles from single PFS nadir spectra in the 1-0 band. They have shown that, even if the retrieved profiles are not vertically resolved, PFS spectra are mostly sensitive to CO in the near-surface atmosphere and, more especially, carry information on the $\mathrm{CO}$ abundance 
below $20 \mathrm{~km}$. These PFS nadir observations could therefore complement NOMAD and ACS solar occultation measurements.

The work of Bouche et al. (2019) is limited to daytime PFS observations. Night-time PFS measurements have indeed been discarded from the analysis due to the strong selection criteria applied to keep only single spectra with sufficient signal-to-noise ratio (SNR). Night-time PFS spectra might however be very useful for the sounding of $\mathrm{CO}$ in the near-surface atmosphere. They are generally associated to large thermal inversions that have been shown to increase the sensitivity of thermal infrared (TIR) measurements to the very near-surface atmosphere but also to slightly increase the vertical resolution of such measurements (Bauduin et al., 2017). A good knowledge of these thermal inversions is therefore essential for the $\mathrm{CO}$ retrieval. We have demonstrated in a companion paper (Bauduin et al., 2020) that this could be achieved by retrieving the atmospheric temperature profile using the information from the $\nu_{3} \mathrm{CO}_{2}$ band (centered at $2349 \mathrm{~cm}^{-1}$ ). The present paper has two main objectives. It aims first at demonstrating that the $\mathrm{CO}$ abundance can be retrieved from night-time nadir PFS spectra and at specifying under which conditions (specific regions and/or periods) this is best done. The second objective of this work is to investigate the potential gain in vertical sensitivity/resolution these observations can provide compared to daytime observations. For this, the paper has been divided in two main sections. Section 2 is related to the detection of $\mathrm{CO}$ and describes the spectral averages that have been necessary to reach sufficient SNR to detect CO. A detection method is also presented. Section 3 focuses on the retrieval of CO profiles from the averaged spectra. It presents the retrieval method and 
settings, the characterization of the retrieved CO profiles in terms of error, vertical sensitivity and information content, and the $\mathrm{CO}$ distribution built from successful night-time retrievals. Section 4 gives the conclusions.

\section{Detection of $\mathrm{CO}$ from night-time observations}

As already mentioned, the challenge of measuring $\mathrm{CO}$ using night-time PFS observations is the very low SNR of this kind of observations in the 5 micron region. However, by making proper averages, it is possible to increase the SNR such that the detection of $\mathrm{CO}$ lines becomes possible (see also Giuranna et al., 2019a, Figure S4 of the supplementary material). In this section, we describe how the averages are performed and present the method set up to assess if $\mathrm{CO}$ is detected in the averaged spectra.

\subsection{Observations and averages}

The observations used in this work are those recorded by the PFS instrument (for details about the instrument and the calibration, see (Formisano et al., 2005; Giuranna et al., 2005a,b)). Since the beginning of its science operation, PFS has collected a large ensemble of Martian nadir spectra, with a dataset covering today 8 full Martian Years (27 MY to $34 \mathrm{MY}$ ) and including about four million of observations in total (including deep space and limb measurements). Among these, we have considered only nadir nighttime observations, which have been defined as the observations for which the solar incidence angle is higher than $90^{\circ}$. As the analysis focuses on the fundamental 1-0 band of $\mathrm{CO}$ (centered at $2143 \mathrm{~cm}^{-1}$ ), only spectra recorded by the shortwave channel (SWC) of PFS have been considered. To simplify the radiative transfer analysis by neglecting the scattering by dust and ice 
particles, PFS observations associated with dust and ice optical depths larger than 0.2 have been discarded. These 2 parameters were provided as part of the PFS level 2 (L2) by the National Institute of Astrophysics INAF-IAPS (Wolkenberg et al., 2018; Giuranna et al., 2019b). In total, 161295 night-time spectra have been selected.

Using this set of night-time observations, averaged spectra have been built. The difficulty has been to keep the averaged spectra the most representative of the Martian atmosphere at the place and time they are associated with, while averaging a sufficiently large number of observations to reach a high enough SNR. Since CO exhibits stronger seasonal and latitudinal variations than longitudinal, we have limited the averages to small solar longitude $\left(\mathrm{L}_{\mathrm{s}}\right)$ periods and to small latitudinal ranges, but we have put no criterion on the longitude. Specifically, the averages have therefore been made for boxes of $10^{\circ}$ of $\mathrm{L}_{\mathrm{s}}$ and $5^{\circ}$ of latitude over the $8 \mathrm{MYs}$, leading to $1163^{1}$, averaged spectra in total.

Figure 1.a shows three examples of averaged spectra for which $\mathrm{CO}$ is detected. The three have low noise and clearly shows the entire band of $\mathrm{CO}$ in emission. $\mathrm{CO}_{2}$ Q-branches are also well identified in emission. The fact that emission features dominate is explained by the presence of thermal inversions, as shown in Figure 1.b. These thermal inversions seem to occur for most of the PFS night-time observations.

\footnotetext{
${ }^{1}$ Some boxes do not include averaged spectra, due to some of the selection criteria.
} 

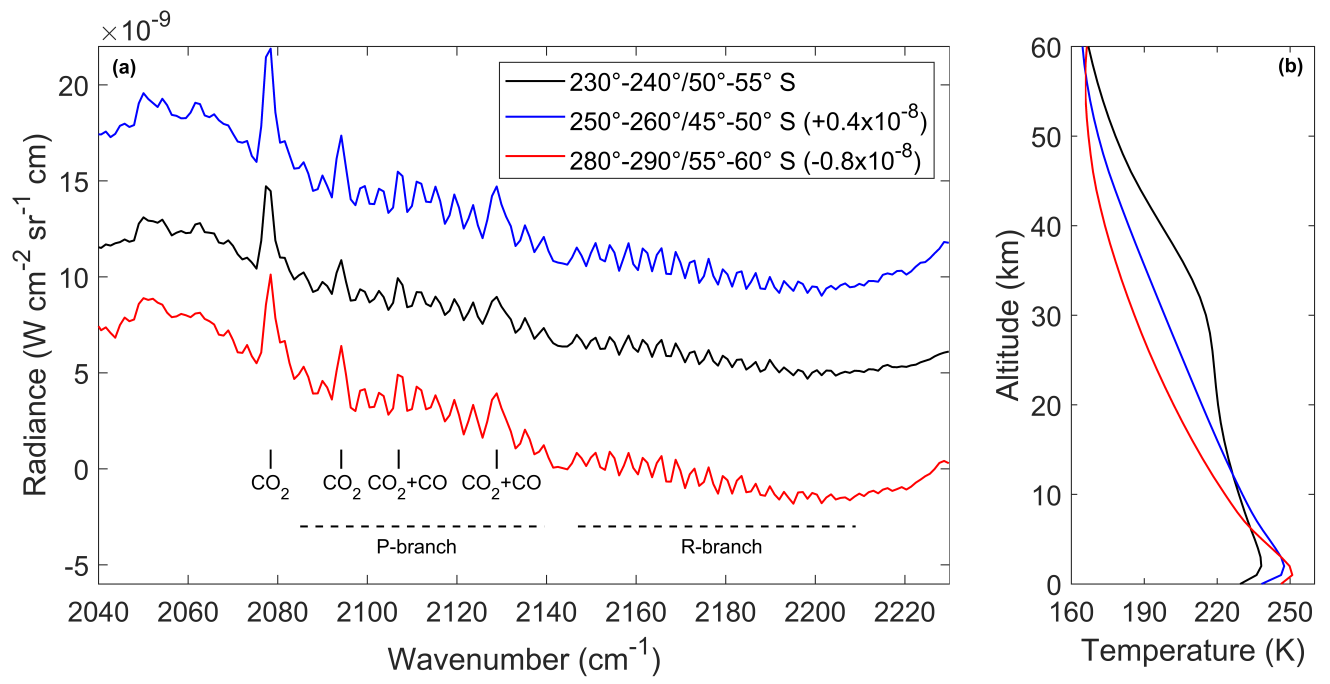

Figure 1: (a) Examples of night-time averaged PFS spectra. For display purposes, spectra have been offset vertically as indicated. The $\mathrm{L}_{\mathrm{s}} /$ latitude boxes to which they correspond are indicated. Some $\mathrm{CO}_{2}$ lines have been identified, along with the P-and R-branches of the CO band. (b) Temperature profiles associated with the three spectra of panel (a). They have been retrieved following the method described in the companion paper (Bauduin et al., 2020).

\subsection{Detection method}

$\mathrm{CO}$ is unfortunately not detected for the 1163 averaged spectra as favourably as in the three example spectra of Figure 1. Its detection is notably impacted by different parameters, such as the surface temperature, which directly affects the SNR, and the thermal contrast, which determines the sensitivity to the near-surface atmosphere in the TIR. To evaluate the observation frequency of $\mathrm{CO}$ in the ensemble of averaged spectra and its dependence on these two parameters, a detection method has been set up. It is based on the estimation of $\mathrm{CO}$ lines intensities using brightness temperature differences (BTD). These differences are calculated between the BT of one target chan- 


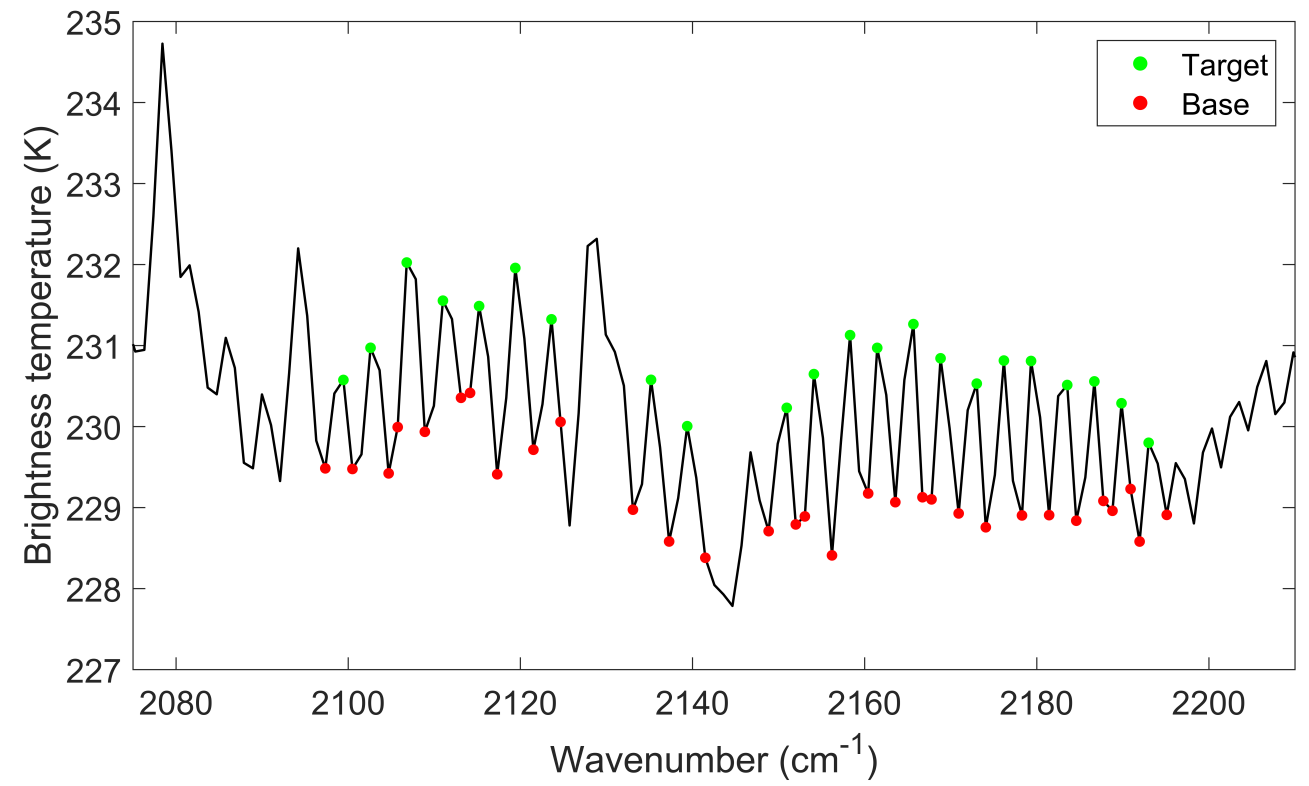
two base channels, representing the baseline of the spectrum and located on each side of the CO line. BTD have been calculated for 21 lines indicated in Figure 2 (the lines are identified with green dots and the base channels correspond to red dots). The BTDs are positive for the lines in emission and negative for lines in absorption.

Figure 2: Spectral channels chosen to calculate the BTD for each CO lines. The channels corresponding to the CO lines (target) are shown in green, and the channels used for the baseline (base) are shown in red. Note that the displayed spectrum is the one corresponding to the box $270^{\circ}-280^{\circ} / 40^{\circ}-45^{\circ} \mathrm{S}$ ( $\mathrm{L}_{\mathrm{s}} /$ latitude).

nel, measured at the center of a CO line, and the averaged BT calculated for 
the $2000-2200 \mathrm{~cm}^{-1}$ range from the following equation:

$$
N E D T_{\tilde{\nu}}=\frac{1}{L_{\tilde{\nu}}}\left(\frac{k_{B} T^{2}}{h c \tilde{\nu}}\right)\left(1-\exp \left(\frac{-h c \tilde{\nu}}{k_{B} T}\right)\right) N E D L_{\tilde{\nu}}
$$

where $N E D L_{\tilde{\nu}}$ is the radiance noise of the averaged spectrum ${ }^{2}, L_{\tilde{\nu}}$ is the radiance at wavenumber $\tilde{\nu}, h$ is the Planck constant, $c$ is the light speed, $k_{B}$ is the Boltzmann constant, and $T$ is the reference temperature, which has been set to $280 \mathrm{~K}$. An additional criterion has been necessary to remove some spectra with noise spikes: spectra with a mean BTD lower than the BTD standard deviation are rejected.

Figure 3 illustrates the detection method applied on the 1163 averaged spectra. Figure 3.a shows the averaged NEDT (over 2000-2200 $\mathrm{cm}^{-1}$ ) as a function of thermal contrast ${ }^{3}$ and surface temperature while Figure 3.b shows the mean BTD (for the $21 \mathrm{CO}$ lines) as a function of these same two parameters. The filled circles correspond to spectra with detectable $\mathrm{CO}$ and the empty circles correspond to those with no CO detection. From Figure 3, the impact of thermal contrast and surface temperature on the detection of $\mathrm{CO}$ is clear. Most detections are, interestingly, associated with large negative thermal contrasts (lower than $-20 \mathrm{~K}$ ), which increase the sensitivity of PFS to near-surface CO, and with large surface temperatures (above $180 \mathrm{~K}$ ), which increase the SNR. Most of the non detections correspond on the contrary to observations with low thermal contrast (between 0 and $-10 \mathrm{~K}$ ) and low surface temperatures (around $145 \mathrm{~K}$ ). For some cases, despite the large thermal

\footnotetext{
${ }^{2}$ To calculate the noise for the averaged spectrum, we have divided the radiance noise of single PFS spectra by the square root of the number of spectra averaged.

${ }^{3}$ The thermal contrast is calculated as the difference between the surface temperature and the mean air temperature of the $0-2 \mathrm{~km}$ layer.
} 

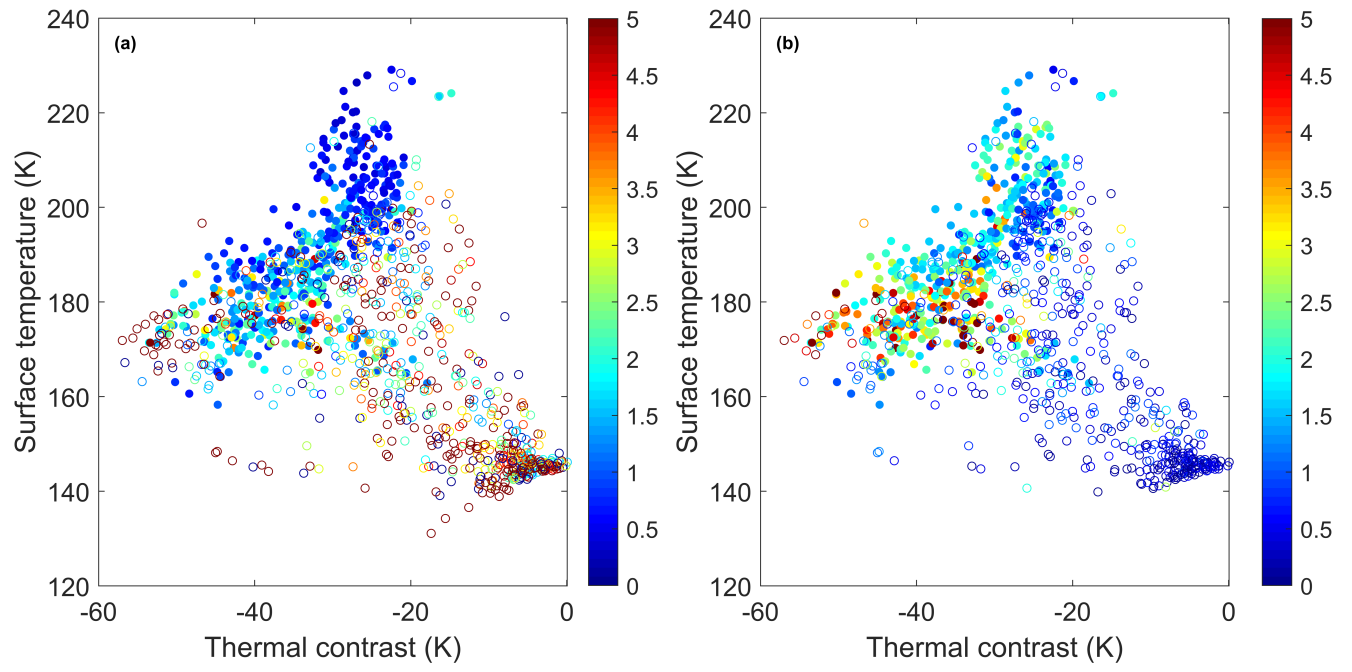

Figure 3: (a) Average NEDT over the $2000-2200 \mathrm{~cm}^{-1}$ range (K, color scale) and (b) mean BTD calculated for the $21 \mathrm{CO}$ lines (K, color scale) as a function of thermal contrast and surface temperature. The filled circles correspond to spectra with detectable $\mathrm{CO}$ and the empty circles to those with a too large noise. The surface temperature associated to each average spectrum has been calculated as the average of the surface temperatures corresponding to single spectra and provided as part of the L2 by INAF-IAPS. The thermal contrast has been calculated as the difference between the surface temperature and the temperature of the air for the $0-2 \mathrm{~km}$ column.

contrast and surface temperature, the mean BTD is significantly lower than the NEDT leading to a non CO detection. In total, 449 spectra over the 1163 present $\mathrm{CO}$ lines emerging from the noise and these have been selected for the following analysis. Interestingly, these selected observations are mainly located in the Southern Hemisphere during spring/summer $\left(180^{\circ}<\mathrm{L}_{\mathrm{s}}<\right.$ $360^{\circ}$ ), where the favourable conditions for the $\mathrm{CO}$ detection (high negative thermal contrast and high surface temperature) are found. This will be discussed in the next section. 


\section{Retrieval of $\mathrm{CO}$ from night-time observations}

In this section, the $\mathrm{CO}$ abundance is retrieved from the 449 averaged spectra selected in the previous section. More particularly, vertical CO profiles are adjusted. Although the vertical resolution of PFS is limited, this allows to take into account the vertical sensitivity of the instrument in the estimation of the $\mathrm{CO}$ column density. More importantly, thanks to the presence of large thermal inversions, the vertical resolution of the $\mathrm{CO}$ profiles could be higher than for daytime observations (Bouche et al., 2019), as it has been shown for Earth nadir TIR spectra (Bauduin et al., 2017). This is discussed in section 3.2 after the description of the retrieval method applied (section 3.1). The distribution of $\mathrm{CO}$ abundance is then presented (subsection 3.3).

\subsection{Retrieval methodology}

\subsubsection{Background}

The retrieval method used to retrieve CO vertical profiles is the Optimal Estimation (OE) (Rodgers, 2000). The idea of the method is to find the $\mathrm{CO}$ profile that is the most consistent with both the averaged night-time spectrum and the prior knowledge of the Martian atmosphere. This is done by minimizing a cost function $J$, written as:

$$
J=(\mathbf{y}-\mathrm{F}(\mathbf{x}, \mathbf{b}))^{\mathrm{T}} \mathbf{S}_{\boldsymbol{\epsilon}}^{-\mathbf{1}}(\mathbf{y}-\mathrm{F}(\mathbf{x}, \mathbf{b}))+\left(\mathbf{x}-\mathbf{x}_{\mathbf{a}}\right)^{\mathrm{T}} \mathbf{S}_{\mathbf{a}}^{-\mathbf{1}}\left(\mathbf{x}-\mathbf{x}_{\mathbf{a}}\right),
$$

where $\mathbf{x}$ is the atmospheric state vector, including the parameters to retrieve (e.g. CO profile, see subsection 3.1.2), $\mathbf{b}$ includes the parameters affecting the top of the atmosphere (hereafter TOA) radiance but that are kept fixed, $\mathbf{y}$ is the vector of measured radiances, $\mathbf{x}_{\mathbf{a}}$ is the a priori state vector, including 
the knowledge of the retrieved parameters before the measurement is made, $\mathbf{S}_{\mathbf{a}}$ is the associated a priori covariance matrix, $\mathbf{S}_{\epsilon}$ is the measurement error covariance matrix associated to $\mathbf{y}$ and $\mathrm{F}$ is the radiative transfer forward model. The minimization of $J$ is performed by a Gauss-Newton iterative scheme, with a new state vector evaluated at each iteration $i$ as follow:

$$
\mathbf{x}_{i+1}=\mathbf{x}_{\mathbf{a}}+\left(\mathbf{K}_{i}^{\mathrm{T}} \mathbf{S}_{\boldsymbol{\epsilon}}^{-1} \mathbf{K}_{i}+\mathbf{S}_{\mathbf{a}}{ }^{-\mathbf{1}}\right)^{-1} \mathbf{K}_{i}^{\mathrm{T}} \mathbf{S}_{\boldsymbol{\epsilon}}^{-\mathbf{1}}\left[\mathbf{y}-\mathrm{F}\left(\mathbf{x}_{i}, \mathbf{b}\right)+\mathbf{K}_{i}\left(\mathbf{x}_{i}-\mathbf{x}_{\mathbf{a}}\right)\right],
$$

with $\mathbf{K}$ the Jacobians matrix, whose rows are the derivatives of the TOA radiance with respect to the retrieved parameters. For more details about the method, see (Rodgers, 2000).

The OE offers an adequate framework to characterize the retrieved profiles in terms of errors and vertical sensitivity. In this work, the retrieval errors are estimated from a total error covariance matrix $\left(\mathbf{S}_{\text {tot }}\right)$, whose the square root of the diagonal elements provide the total retrieval error for each retrieved layer. This matrix is calculated as follows (Rodgers, 2000)

$$
\mathrm{S}_{\mathrm{tot}}=\mathrm{S}_{\mathrm{s}}+\mathrm{S}_{\mathrm{m}}+\mathrm{S}_{\mathbf{f}}
$$

where $\mathbf{S}_{\mathbf{s}}$ is the covariance matrix of the smoothing error, which characterizes the uncertainty on the $\mathrm{CO}$ profile due to the limited vertical resolution of nadir PFS observations, $\mathbf{S}_{\mathbf{m}}$ is the covariance matrix of the measurement error, which characterizes the uncertainty on the $\mathrm{CO}$ profile due to the measurement noise, and $\mathbf{S}_{\mathbf{f}}$ is the covariance matrix of the fitted parameters error, taking into account the interferences between the retrieved $\mathrm{CO}$ profile and the rest of the state vector (here, surface temperature and $\mathrm{CO}_{2}$ profile, see section 3.1.2). These three matrices are calculated using the following 
equations (Rodgers, 2000):

$$
\begin{aligned}
\mathbf{S}_{\mathbf{s}} & =(\mathbf{A}-\mathbf{I}) \mathbf{S}_{\mathbf{a}}(\mathbf{A}-\mathbf{I})^{\mathrm{T}} \\
\mathbf{S}_{\mathbf{m}} & =\mathbf{G ~ S}_{\boldsymbol{\epsilon}} \mathbf{G}^{\mathrm{T}} \\
\mathbf{S}_{\mathbf{f}} & =\mathbf{A}_{\mathrm{ir}} \mathbf{S}_{\mathbf{r a}} \mathbf{A}_{\mathrm{ir}}^{\mathrm{T}}
\end{aligned}
$$

where $\mathbf{A}$ is the averaging kernels matrix (see below), $\mathbf{I}$ is the identity matrix, $\mathbf{G}$ is the gain matrix, whose rows are the derivatives of the retrieved state with respect to the measurement, $\mathbf{A}_{\mathbf{i r}}$ are the non-diagonal blocs of the averaging kernels matrix, $\mathbf{i}$ is the part of the state vector under consideration (i.e. the $\mathrm{CO}$ profile), $\mathbf{r}$ represents the rest of the state vector (i.e. the surface temperature and the $\mathrm{CO}_{2}$ profile), and $\mathbf{S}_{\mathrm{ra}}$ is the a priori covariance matrix of $\mathbf{r}$. The vertical sensitivity and resolution of the retrieval is described by the averaging kernels (AVK) functions. They are defined as the rows of the AVK matrix (A), calculated as

$$
\mathbf{A}=\left(\mathbf{K}^{\mathrm{T}} \mathbf{S}_{\epsilon}^{-1} \mathbf{K}+\mathbf{S}_{\mathbf{a}}^{-1}\right)^{-1} \mathbf{K}^{\mathrm{T}} \mathbf{S}_{\epsilon}^{-1} \mathbf{K} .
$$

One AVK function is associated to $\mathrm{CO}$ in each retrieved layer. It peaks at the altitude from where most of the information about $\mathrm{CO}$ in that layer is coming. The AVKs thus provide an estimation of the altitude of maximum sensitivity to CO. Their width gives also an estimation of the retrieval vertical resolution. The trace of the matrix $\mathbf{A}$ corresponds to the degrees of freedom for signal (DOFS) of the retrieval.

The AVK matrix allows expressing the retrieved state $(\hat{\mathbf{x}})$ as a function of the true $(\mathbf{x})$ and a priori states (if we ignore the term involving random errors) (Rodgers, 2000):

$$
\hat{\mathbf{x}}=\mathbf{A x}+(\mathbf{I}-\mathbf{A}) \mathbf{x}_{\mathbf{a}}
$$


with I the identity matrix. The retrieved state at any vertical location is thus a combination between: 1) an average of the true profile weighted by the corresponding AVK function and 2) an average of the a priori profile weighted by $(\mathbf{I}-\mathbf{A})$. To visualize more easily the impact of the a priori profile on the retrieved state, it is useful to perform an eigenvector decomposition of the matrix A (Rodgers, 2000). If the matrix $\mathbf{R}$ is the matrix of the right eigenvectors of $\mathbf{A}$, we can write

$$
\mathrm{AR}=\mathbf{R} \boldsymbol{\Lambda}
$$

where $\Lambda$ is a diagonal matrix, whose diagonal elements correspond to the eigenvalues of $\mathbf{A}$. If we multiply each side of equation 9 by $\mathbf{R}^{-\mathbf{1}}$ and using equation 10 , we obtain

$$
\begin{aligned}
\mathbf{R}^{-1} \hat{\mathbf{x}} & =\mathbf{R}^{-1} \mathbf{A x}+\mathbf{R}^{-1}(\mathbf{I}-\mathbf{A}) \mathbf{x}_{\mathbf{a}} \\
& =\Lambda \mathbf{R}^{-1} \mathbf{x}+(\mathbf{I}-\mathbf{\Lambda}) \mathbf{R}^{-1} \mathbf{x}_{\mathbf{a}}
\end{aligned}
$$

In the eigenvectors of $\mathbf{A}$ basis, the vector $\mathbf{x}$ becomes $\mathbf{R}^{-1} \mathbf{x}=\mathbf{z}$ and thus, we have $\mathbf{x}=\mathbf{R z}$. The vector $\mathbf{x}$ (and so the retrieved, the true and the $a$ priori states) is therefore represented as a linear combination of the right eigenvectors of $\mathbf{A}$, and $\mathbf{z}$ is the coefficients vector. Using these relations, equation 11 becomes

$$
\hat{\mathbf{z}}=\Lambda \mathbf{z}+(\mathbf{I}-\mathbf{\Lambda}) \mathbf{z}_{\mathbf{a}}
$$

Because $\boldsymbol{\Lambda}$ is diagonal, the above equation can be separated into scalar components such that

$$
\hat{z}_{i}=\lambda_{i} z_{i}+\left(1-\lambda_{i}\right) z_{a i}
$$

Considering that all profiles are decomposed into a combination of patterns (eigenvectors of $\mathbf{A}$ ), the retrieved coefficients $\hat{z}_{i}$ are a weighted average of 
the true $z_{i}$ and a priori $z_{a i}$ coefficients. The patterns associated to $\lambda_{i} \sim 1$ are dominated by the true state, and thus well measured, while those associated to $\lambda_{i} \sim 0$ are determined by the a priori state. Note that we have not considered the measurement error in the above mathematical development. It is important to keep in mind that in reality a third term including the random uncertainties of the measurement also impacts the value of the retrieved coefficients $\hat{z}_{i}$. Finally, it can be shown that the DOFS is the sum of the eigenvalues of $\mathbf{A}$. It corresponds therefore to the number of components/patterns in the retrieved profile that are measured independently of the a priori.

\subsubsection{Retrieval settings and input to the radiative transfer}

The analysis has been divided in two steps: 1) the retrieval of the temperature profile, and 2) the retrieval of the CO profile. The retrieval of the temperature profile has been performed on the $\nu_{3}$ band of $\mathrm{CO}_{2}$ centered at $2349 \mathrm{~cm}^{-1}$ for each averaged night-time spectrum. The details about this retrieval, the choice of its settings and the associated error characterization are fully described in the companion paper (Bauduin et al., 2020) and are not reminded hereafter. The retrieved temperature profiles are then used as input for the $\mathrm{CO}$ retrieval. Before going further, it has to be pointed out that the temperature profile retrieval was successful for 350 out of the 449 averaged spectra (for 99 observations, the temperature retrievals have diverged, i.e. a temperature profile could not be retrieved for these observations). Only for these 350 spectra a CO profile retrieval was performed. More particularly, the CO retrieval has been performed on the $2040-2230 \mathrm{~cm}^{-1}$ spectral range. The CO a priori profile and covariance matrix are those used by Bouche 
et al. (2019). As a reminder, they have been built from a large ensemble of profiles modelled by the MCD (version 5.3) for one Martian year, using the climatology solar average scenario. In total, 451584 modelled CO profiles have been used. The resulting $\mathbf{x}_{\mathbf{a}}$ is shown in Figure 4.b along with the $a$ priori uncertainties (error bars), which correspond to the square root of the diagonal elements of the $\mathbf{S}_{\mathbf{a}}$. The atmosphere used extends from 0 to $60 \mathrm{~km}$. The temperature profile considered is the one retrieved in the first step. CO is retrieved for $2 \mathrm{~km}$ thick layers from 0 to $24 \mathrm{~km}$ (Bouche et al., 2019). Above that altitude and up to $60 \mathrm{~km}$, the $\mathrm{CO}$ profile is kept fixed to the a priori. As shown in Figure 1.a, $\mathrm{CO}_{2}$ presents strong $\mathrm{Q}$ branches in the spectral range considered, some of which interfere with CO lines. This gas has thus been retrieved simultaneously with $\mathrm{CO}$ only to obtain the best fit for CO. As for the latter, $\mathrm{CO}_{2}$ has been retrieved for $2 \mathrm{~km}$ thick layers from 0 to $24 \mathrm{~km}$, and then fixed to the a priori profile above that level. This $\mathrm{CO}_{2}$ a priori profile is the same as the one used to retrieve the temperature profile and corresponds to a global average calculated from $\mathrm{CO}_{2}$ profiles modelled by the MCD (version 5.3), with same scenario as for $\mathrm{CO}$. These modelled $\mathrm{CO}_{2}$ profiles have also been used to build the associated a priori covariance matrix (Bouche et al., 2019). Note that the retrieval of $\mathrm{CO}_{2}$ is strongly constrained (maximal a priori variability of $2 \%$ at the surface) and leads to only small variations of surface pressure ( 5 times smaller than the uncertainty considered in our error calculations, see section 3.2.4). Note also that ozone $\left(\mathrm{O}_{3}\right)$ and water vapour $\left(\mathrm{H}_{2} \mathrm{O}\right)$ have absorption bands in the spectral range considered. Although their contributions are negligible with no visible spectral features, we have fixed their abundance to climatological modelled profiles (built from the 
$\mathrm{MCD}$, version 5.3). In addition to $\mathrm{CO}$ and $\mathrm{CO}_{2}$, the surface temperature has been retrieved to properly model the baseline of the spectrum. The a priori value was set to the averaged surface temperature calculated from the L2 surface temperatures associated to each spectrum considered in the spectral average. The a priori variability has been set to $1 \%$. For the emissivity, an average value for each average spectrum has been calculated by co-localizing single spectra with the emissivity at $2000 \mathrm{~cm}^{-1}$ global maps provided by the Planetary SUrface Portal (Poulet et al., 2018; Quantin-Nataf et al., 2018). It has been kept fixed during the retrieval. Finally, the spectroscopic parameters have been taken from HITRAN 2016 (Gordon et al., 2017), including the pressure broadening by $\mathrm{CO}_{2}$, and the instrumental line shape recommended by the PI team of PFS has been considered (Giuranna et al., 2019a).

All retrievals have been performed with the Atmosphit software (Barret et al., 2005; Coheur et al., 2005). This is a line-by-line radiative transfer code initially developed for Earth atmospheric spectra, but which has been successfully adapted to analyse PFS Martian observations (Bouche et al., 2019). Note that when performing the retrieval of an abundance profile, Atmosphit adjusts a vector of scaling factors, that applied on the a priori profile gives the retrieved profile. In the case of our retrieval, partial columns of $\mathrm{CO}$ are first calculated for the retrieved layers by applying the retrieved scaling factors onto the a priori partial columns. This is done for $\mathrm{CO}_{2}$ as well. Then, to obtain the profiles expressed in volume mixing ratios (VMR), the air partial columns are calculated using the retrieved $\mathrm{CO}_{2}$ partial columns, but also including the retrieved $\mathrm{CO}$ partial columns and the fixed $\mathrm{H}_{2} \mathrm{O}$ and $\mathrm{O}_{3}$ columns, determined from the chosen climatological profiles. Abundance 
of nitrogen $\left(\mathrm{N}_{2}\right)$ and argon (Ar) are also taken into account, assuming mole fractions respectively of 0.027 and 0.016 .

\subsection{Characterization of retrieved $C O$ profiles}

In this section, we focus on the characterization of the retrieved CO profiles in terms of vertical sensitivity and errors. Among the 350 spectra analysed, only 170 have been successfully fitted (i.e. the root mean square of the residual is similar to the noise level considered). The characterization is discussed only for these successful retrievals and is especially illustrated with one chosen case, which corresponds to an average PFS spectrum located in the Southern Hemisphere (in the box $\left.40^{\circ} \mathrm{S}-35^{\circ} \mathrm{S}\right)$ during the summer $\left(280^{\circ}<\right.$ $\mathrm{L}_{\mathrm{s}}<290^{\circ}$ ). It is shown in Figure 4.a along with the resulting fit. The root mean square (RMS) of the residual is $1.74 \times 10^{-10} \mathrm{~W} \mathrm{~cm}^{-2} \mathrm{sr}^{-1} \mathrm{~cm}$ and is of the same order as the NEDL estimated to be $1.87 \times 10^{-10} \mathrm{~W} \mathrm{~cm}^{-2} \mathrm{sr}^{-1} \mathrm{~cm}$. In the following subsections, we present the characterization in terms of the retrieved profile and its associated errors (section 3.2.1), of the vertical sensitivity (section 3.2.2) and of the a priori impact (section 3.2.3).

\subsubsection{Retrieved profile and errors}

The retrieved CO profile for the chosen example is shown in Figure 4.b with the corresponding errors for each retrieved layers. The largest VMR is retrieved for the first layer $(0-2 \mathrm{~km})$ and is $798 \mathrm{ppm}$. It then decreases to around $640 \mathrm{ppm}$ at $5 \mathrm{~km}$ and stays almost constant up to the highest retrieved layer. The column-averaged VMR calculated for the range 0-24 km is equal to $668 \mathrm{ppm}$. Regarding the errors, the lowest error is found at the surface. It is around $10 \%$ and this corresponds to a large reduction of the 

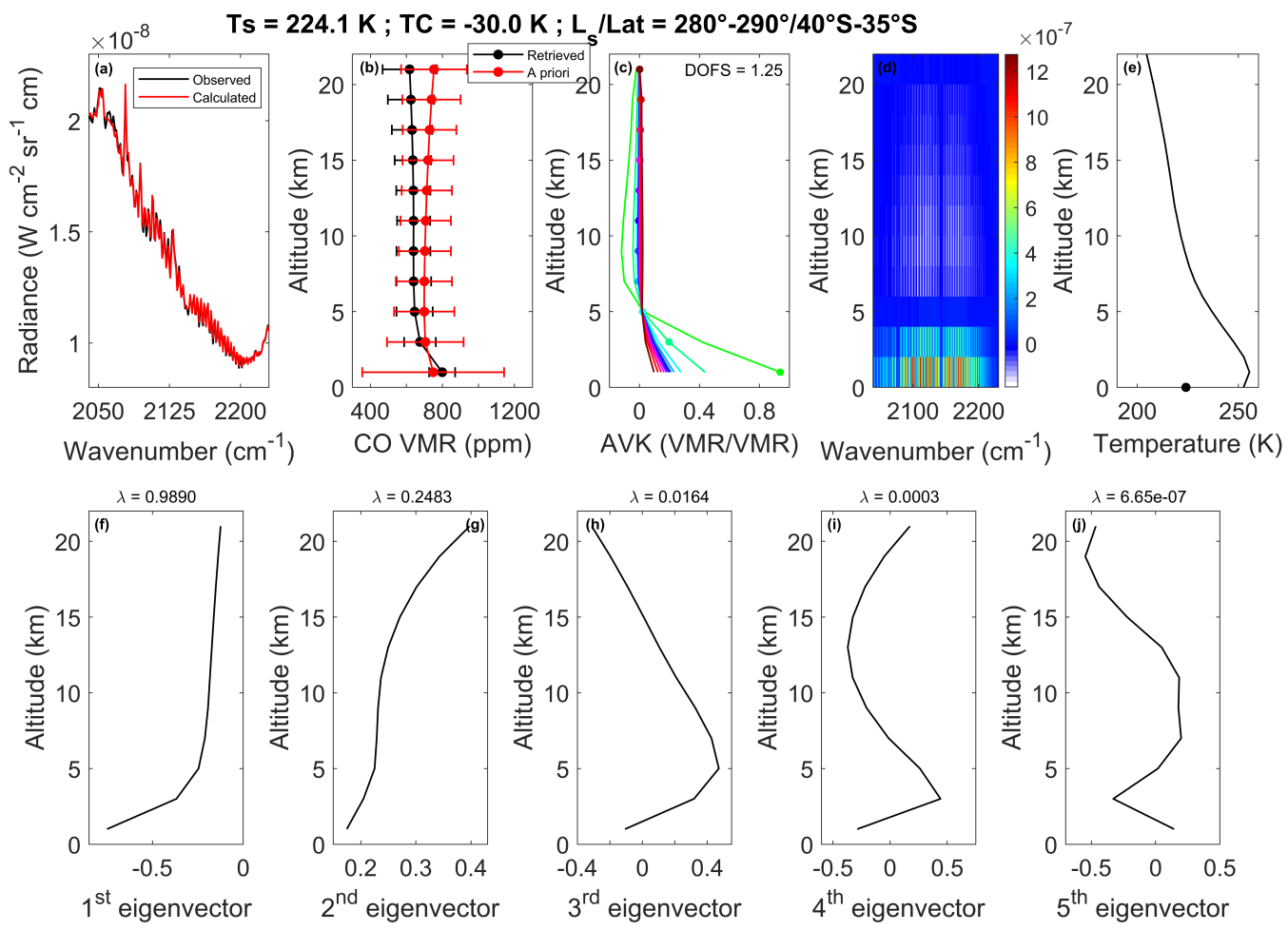

Figure 4: Characterization of the $\mathrm{CO}$ retrieved profiles for a representative retrieval. (a) Resulting fit of the spectrum. (b) Retrieved CO profile (black) and a priori profile (red) expressed in volume mixing ratios (ppm). (c) Averaging kernels functions for the CO retrieved profile expressed in VMR units. They provide an estimation of the altitude of maximum sensitivity to CO. The dots indicate to which layer the AVK functions correspond. (d) Jacobians ( $\mathrm{W} \mathrm{cm} \mathrm{cm}^{-2} \mathrm{sr}^{-1} \mathrm{~cm}$ per VMR, color scale) calculated for the retrieved layers. (e) Retrieved temperature profile (Bauduin et al., 2020) and surface temperature (black dot). (f-j) The first five eigenvectors of the AVK matrix and their associated eigenvalues $(\lambda)$, allowing to evaluate the impact of the a priori profile on the retrieved $\mathrm{CO}$ profile (see text for details). $3 \mathrm{~km}$ and stays constant up to $15 \mathrm{~km}$. Above that layer, it increases again to 


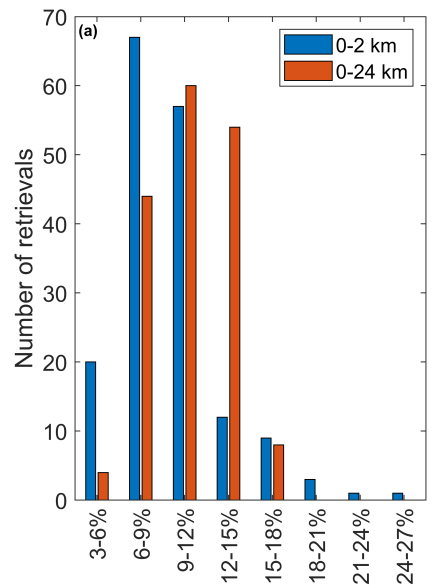

more than $20 \%$. The error on the total column $(0-24 \mathrm{~km})$ is estimated to be around $14 \%$. Note that this is not the total error. Additional contributions due to uncertainties on the temperature profile, surface pressure and ILS formulation will be estimated in section 3.2.4.
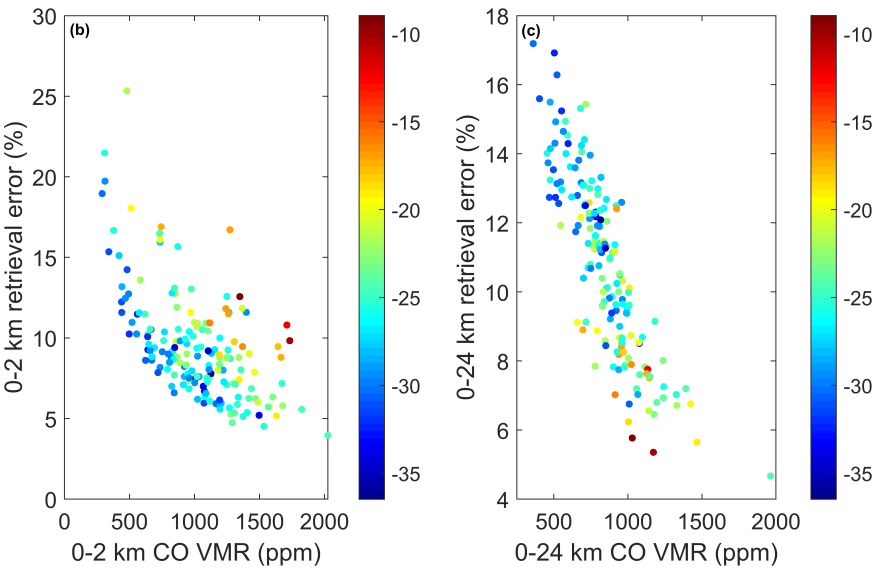

Figure 5: (a) Histogram of the number of retrievals as a function of the total retrieval error (\%) on the 0-2 km CO column (blue bars) and on the 0-24 km CO column (orange bars). (b) Dependence of the retrieval error on the 0-2 km CO VMR (\%) on the CO VMR itself and on the thermal contrast (K, color scale). (c) Same as (b) but for the 0-24 km VMR.

To have a better representation of the retrieval errors for all the analysed observations, Figure 5.a shows the distribution of the errors (for the 0-2 km and 0-24 km CO columns) among the retrieval set. For most of the average spectra analysed (73\%), the retrieval uncertainty on the $0-2 \mathrm{~km}$ column falls in the range $6-12 \%$. For around $12 \%$ of data, the retrieval error on the first layer is smaller than $6 \%$ and for $15 \%$, it is larger than $12 \%$. The retrieval error 
on the total column is very small, between 6 and $15 \%^{4}$, for the large majority of the analysed set (93\%). The cause of the variations of the retrieval error can be investigated with the help of Figures 5.b and 5.c. In the case of the retrieval error on the $0-2 \mathrm{~km}$ column (Figure 5.b), the variations are driven mainly by one parameter: the thermal contrast. Indeed, for a constant 0-2 $\mathrm{km}$ column, the retrieval error increases with a decrease (in absolute value) of the thermal contrast. The latter indeed drives the sensitivity of PFS to the near-surface CO: the larger (in absolute value) the thermal contrast, the higher the sensitivity to the 0-2 km layer, and therefore, the lower the associated retrieval error. This dependency of the errors on the thermal contrast is however much lower for the total $(0-24 \mathrm{~km})$ column retrieval error (Figure 5.c). Note finally that the thermal contrast values sampled in this work are highly negative. Even the lowest values (in absolute) are sufficiently high to allow PFS to have some sensitivity to the near-surface CO. This is shown particularly by the low errors on the surface layer even for the smallest thermal contrasts (absolute value) (see also (Bauduin et al., 2017)). This could explain why the impact of thermal contrast on the total column retrieval error is so small.

\subsubsection{Vertical sensitivity}

For the chosen case, the fact that the lowest error is found in the first layer is explained by the presence of a thermal inversion and by the associated high negative thermal contrast (-30 K, see Figure 4.e), which enhances PFS

\footnotetext{
${ }^{4}$ Note again that this is not the total error. Additional contributions will be estimated in section 3.2.4.
} 
sensitivity towards the $\mathrm{CO}$ close to surface. The maximum sensitivity to $\mathrm{CO}$ is indeed found at the surface as shown by the averaging kernels functions (Figure 4.c) and by the Jacobians (Figure 4.d). The AVK functions for all retrieved layers peak at the surface. This shows that CO retrieved in each layer, even the highest ones, is mostly inferred from the $\mathrm{CO}$ abundance of the first layer. Note especially the very high value of 0.93 at the surface for the AVK of the first layer, showing a very high sensitivity of the retrieved CO VMR in the $0-2 \mathrm{~km}$ layer to the true CO VMR of that same layer. Above the 4-6 km layer, all the AVK functions are very close to 0 (except for the 0-2 $\mathrm{km}$ AVK, see below). This means that the retrieved CO VMR above $6 \mathrm{~km}$ is mainly driven by the $\mathrm{CO}$ abundance of the layers close to the surface. This is also observed with the Jacobians, that show maximal values close to the surface and very low sensitivity between 5 and $15 \mathrm{~km}$ (a factor 6 lower than the sensitivity at the near-surface). It is worth pointing out that the 0-2 $\mathrm{km}$ AVK shows small negative values above $6 \mathrm{~km}$ (minimum of -0.12). These negative values tend to show that there is anti-correlation between the $0-2 \mathrm{~km}$ layer and the layers above $6 \mathrm{~km}$. This can be understood in terms of emission and absorption. Indeed, because of the presence of the thermal inversion (Figure 4.e), emission takes place close to the surface. Above the thermal inversion (above $4 \mathrm{~km}$ ), due to the negative temperature difference between two adjacent layers (i.e. temperature decreases with height), absorption takes place. Therefore, increasing the $\mathrm{CO}$ abundance close to the surface has the same effect on the PFS spectra as decreasing the $\mathrm{CO}$ abundance above the thermal inversion (the contrary is also true), and this explains the observed anti-correlation. However, regarding the difference in PFS sensitivity to the 


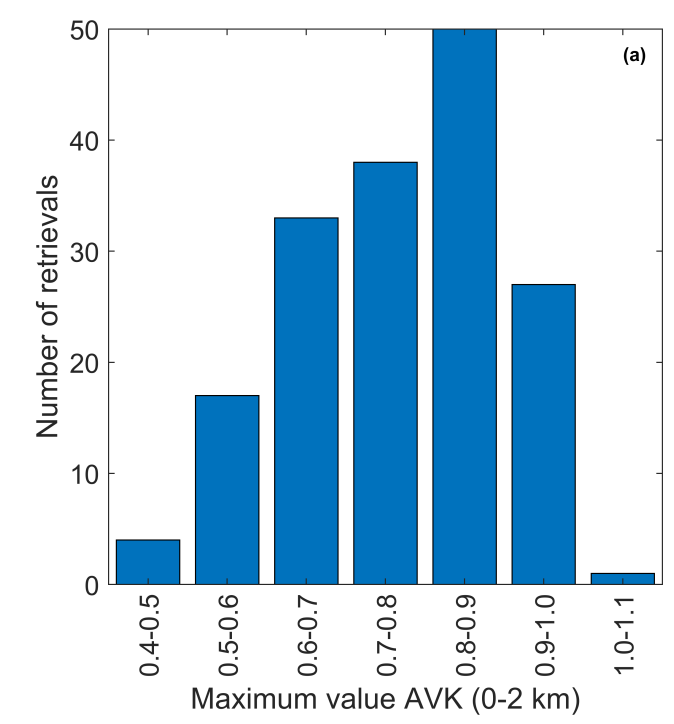
the $0-6 \mathrm{~km}$ region.

two atmospheric regions as shown by the Jacobians (Figure 4.d), the anticorrelation is likely limited. Finally, given the very similar shape of all AVK functions, one has to conclude that the retrieved CO profile is not resolved vertically. The retrieval provides only one column that is mainly sensitive to

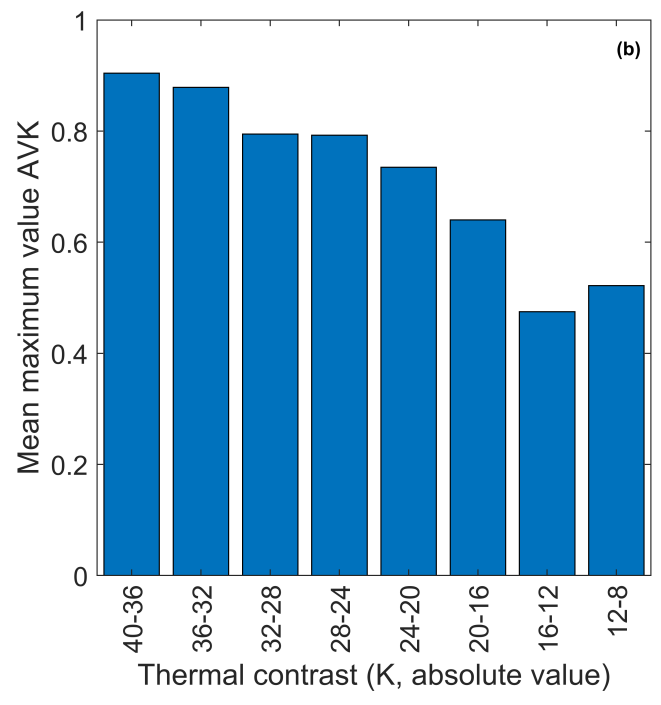

Figure 6: (a) Histogram of the number of $\mathrm{CO}$ retrievals as a function of the maximum AVK value for the $0-2 \mathrm{~km}$ layer. (b) Histogram of the mean maximum AVK value $(0-2$ $\mathrm{km}$ layer) per bin of $4 \mathrm{~K}$ of thermal contrast (shown in absolute value).

For all night-time averaged spectra, the AVKs functions present also very similar shapes, which means that the vertical CO profile is not resolved for any of the retrievals. Because of this lack of vertical resolution, for all retrievals, we use as diagnostic of the sensitivity only the value of the AVK for the $0-2 \mathrm{~km}$ layer. It has indeed the largest values of all AVK functions for all observations and it is the one showing the most clearly the anticorrelation between the surface layers and the higher layers. In fact, the 
anti-correlation remains limited for most of the retrievals, with $66 \%$ of the cases presenting a negative AVK value smaller than 0.1 . Then, by noting that $86 \%$ of the observations have their first AVK peaking in the first layer and $14 \%$ in the second layer, we can safely conclude that for the entire set the maximum sensitivity is close to the surface. The exact location of the maximum depends principally on the temperature profile and on the thermal inversion. Figure 6.a shows the distribution of the maximum value of the 0$2 \mathrm{~km}$ AVK among the analysed set. $68 \%$ of the retrievals present maximum AVK values larger than 0.7, and these are found for the 0-2 km AVK peaking in the first layer. This indicates that for the majority of the analyzed cases, the retrieved $\mathrm{CO}$ abundance in the $0-2 \mathrm{~km}$ is mainly sensitive to the true $\mathrm{CO}$ abundance in that layer. The variations of the maximal value of the $0-2 \mathrm{~km}$ AVK are among other driven by the thermal contrast. Figure 6.b shows the mean maximal AVK value per bin of $4 \mathrm{~K}$ of thermal contrast. The impact of the latter on the maximal value is clear, with maximum value of $0.8-0.9$ found for thermal contrast higher than $30 \mathrm{~K}$ (absolute value) and decreasing to 0.5 for smaller values. Finally, as already mentioned, the $\mathrm{CO}$ vertical profile is not resolved for any of the retrievals. The majority of these provide only one column that is mainly sensitive to the $0-10 \mathrm{~km}$ atmospheric region.

\subsubsection{Impact of the a priori profile}

The DOFS of the example retrieval of Figure 4 is equal to 1.25. This corresponds to the number of patterns in the retrieved profile that are determined independently of the a priori. This can be seen with the eigenvectors decomposition of the matrix $\mathbf{A}$ (reminding that the retrieved profile can be expressed as a linear combination of the eigenvectors of $\mathbf{A}$, section 3.1.1). 


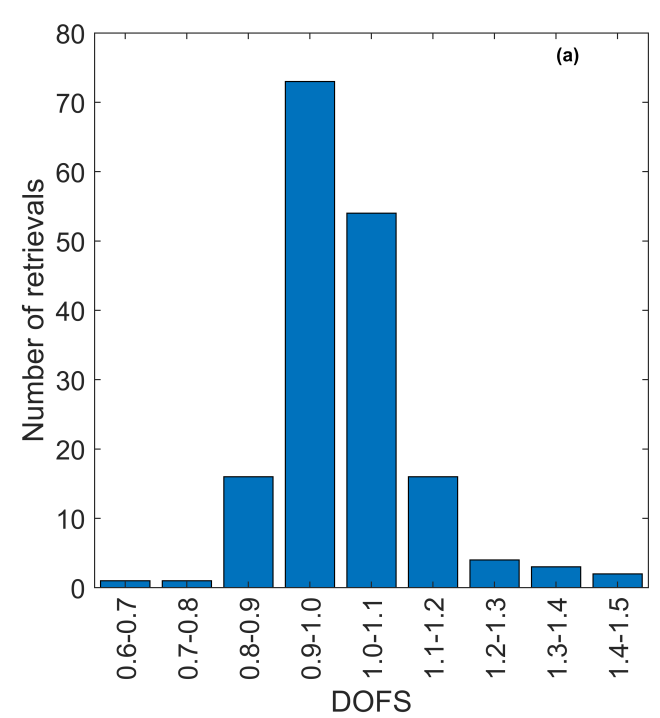

Figure 4 (f-j) shows the first five eigenvectors of $\mathbf{A}$ and the corresponding eigenvalues. The first eigenvector is associated to an eigenvalue of 0.99. This component of the retrieved profile is therefore well measured: its associated coefficient $\hat{z}_{1}$ is determined almost entirely from the true state $\left(z_{1}\right)$. The second eigenvalue is equal to 0.25 . The coefficient associated the second component is thus dominated by the a priori state $\left(z_{a 2}\right)$, and only a small part (around 25\%) is determined from the true state $z_{2}$. The following eigenvectors have finer structures, but their associated eigenvalues reach almost 0 . The coefficients for all these components are all determined from the a priori state, and this confirms the very low vertical resolution of the retrieval.



Figure 7: (a) Histogram of the number of CO retrievals as a function of the DOFS. (b) Same as (a) but as a function of the first (blue) and second (orange) eigenvalues.

Figure 7.a shows the distribution of the DOFS values among all successful retrievals. Almost $90 \%$ present values larger than 0.9, showing that for the large majority of the retrievals, at least one pattern in the retrieved profile is 
well measured, i.e., it is determined independently of the a priori. As can be seen from Figure 7.b, this pattern corresponds to the first eigenvectors (associated eigenvalues within 0.9 and 1.0). However, for $84 \%$ of the retrievals, the second eigenvalue is close to 0 , showing that the other patterns in the retrieved profile are determined from the a priori state. In fact, only a small fraction $(16 \%)$ of the retrievals present a second eigenvalue slightly larger than 0.1 , meaning that the second pattern in the retrieved profile is partly determined from the true state.

\subsubsection{Other sources of errors}

Two error sources have not been included in the error budget described in section 3.2.1 and both come from the propagation of the uncertainties on the retrieved temperature profile. There are two types of uncertainties associated to the temperature profile. The first one includes the smoothing and measurement errors and is calculated from the total error covariance matrix. This has been done in section 4.1 of the companion paper (Bauduin et al., 2020), and we have shown that this error varies between $0.5 \mathrm{~K}$ and $2 \mathrm{~K}$ depending on the altitude. It will be called the temperature retrieval error hereafter. The second one is associated to the uncertainty on the pressure (i.e., $\mathrm{CO}_{2}$ ) profile. Indeed, we have made an assumption for the pressure profile (see (Bauduin et al., 2020)) and any error on it obviously propagates to the retrieved temperature profile and subsequently to the retrieved $\mathrm{CO}$ abundance. This temperature uncertainty (hereafter called temperature-pressure error) has also been estimated in section 4.1 of the first part paper and evaluated to be within $0.5-4 \mathrm{~K}$ (in absolute value) depending on the altitude. These two types of temperature errors have been propagated on the CO retrieval sepa- 
rately but following a similar procedure. For the 170 averaged spectra that we kept, we have redone the $\mathrm{CO}$ retrieval but the temperature profile has been shifted $( \pm)$ by the estimated errors. Note that in the case of the temperature pressure error, the surface pressure has been shifted simultaneously by $\pm 0.9 \mathrm{hPa}$, because these two sources of uncertainty are intimately related (see Bauduin et al. (2020) for details). The CO error due to the temperature pressure error includes therefore the contributions of both the temperature profile and the surface pressure uncertainties. The error on CO has been taken as the difference between the new and the reference $\mathrm{CO}$ abundance. Note that this evaluation is performed only for the column-averaged VMR as the $\mathrm{CO}$ profile is not resolved.

The distributions of the two types of uncertainties among the retrievals are shown in Figure 8.a and Figure 8.b for the propagation of respectively the temperature retrieval error and the temperature-pressure error. For the former (Figure 8.a), the distribution is separately shown for a negative (blue bars) and a positive shift (orange bars) of the temperature profile. In both cases, more than $75 \%$ of the retrievals show that the CO uncertainty due to the temperature retrieval error is below $10 \%$. The average is around $6 \%$ for both cases. Regarding the $\mathrm{CO}$ uncertainty due to the temperature-pressure error (Figure 8.b), its distribution is shown separately for the error coming from a surface pressure shift of $-0.9 \mathrm{hPa}$ (blue bars) and $+0.9 \mathrm{hPa}$ (orange bars). This distribution is similar to the one obtained in Figure 8.a, with the $\mathrm{CO}$ uncertainty due to the temperature-pressure error being on average $6 \%$ and below $10 \%$ for a large majority of the retrievals (more than $80 \%$ ).

It is worth reminding that Bouche et al. (2019) have evaluated the im- 

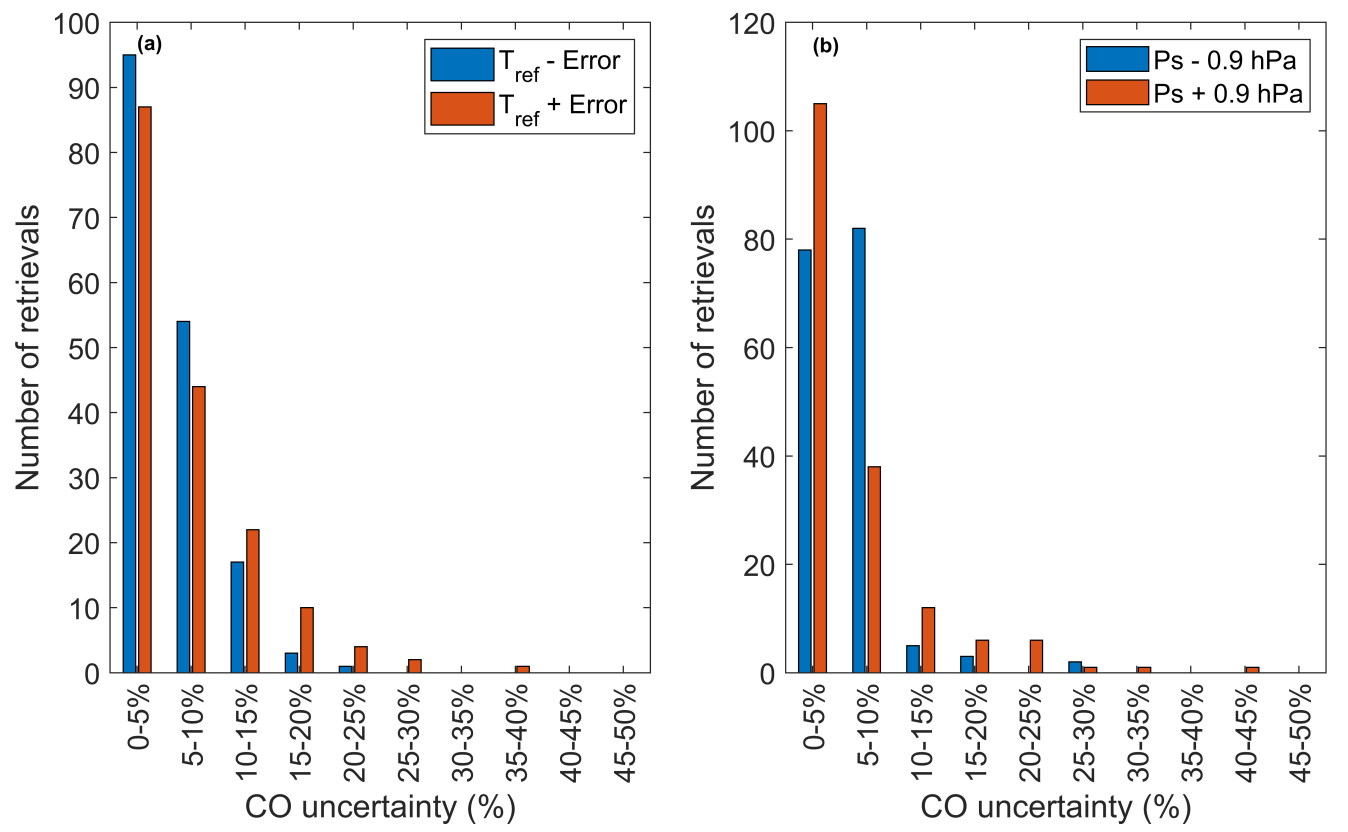

Figure 8: (a) Histogram of the number of $\mathrm{CO}$ retrievals as a function of the $\mathrm{CO}$ uncertainty (absolute value) due to the temperature retrieval error. The blue and orange bars correspond to the $\mathrm{CO}$ uncertainty due respectively to a negative and positive shift of the retrieval error (Bauduin et al., 2020). (b) Same as panel (a) but for the CO uncertainty due to the temperature-pressure error. Note that this error includes the contributions of both the temperature profile and surface pressure uncertainties. The blue and orange bars correspond to the $\mathrm{CO}$ uncertainty due to the temperature error evaluated when shifting the surface pressure respectively of $-0.9 \mathrm{hPa}$ and $+0.9 \mathrm{hPa}$ (see Bauduin et al. (2020)).

pact of the instrumental line shape (ILS) formulation on the retrieved CO abundance. They showed that the uncertainty on the ILS can lead to a systematic uncertainty of around $5 \%$ on the $\mathrm{CO}$ retrieved total column. A re-evaluation of this error has been performed (not shown) and has led to a larger error on $\mathrm{CO}$, of around 14\%. Assuming that all the errors discussed above linearly add up, the total error on the CO 0-24 km column is within 
$32-41 \%$ on average. Note finally that, even if only spectra associated with a dust optical depth lower than 0.2 have been selected, because we have neglected the scattering some additional errors might contribute to the total error on the $\mathrm{CO}$ abundance. However, these errors should be low given the fact that scattering by dust does not contribute significantly to the spectral range considered (Billebaud et al., 2009).

\subsection{Distribution}

Figure 9.a shows the latitudinal distribution of the retrieved $\mathrm{CO}$ columnaveraged VMRs as a function of $\mathrm{L}_{\mathrm{s}}$. As already mentioned in the previous section, only the retrievals with a satisfactory fit (corresponding to a fit with a RMS similar to the noise level) have been kept (170 out of 350), and only these have been used to build the distribution. Also, as a reminder, the detection method (section 2) already rejected a number of spectra: only 449 average spectra out of 1163 were kept and these were mostly observations with $180^{\circ}<\mathrm{L}_{\mathrm{s}}<360^{\circ}$ and located in the Southern Hemisphere. The postfiltering based on the fit quality (for both temperature and $\mathrm{CO}$ retrievals, see previous sections) is further responsible for the lack of $\mathrm{CO}$ measurements in the regions and periods of pre-selected observations.

The mean CO column-averaged VMR calculated for the selected set of observations is $845 \mathrm{ppm}$, which is in good agreement with the averages calculated from daytime measurements (Smith et al., 2018; Bouche et al., 2020). As seen in Figure 9.a, the retrieved CO VMRs distribution is noisy, especially in the equatorial and northern mid-latitude regions. The individual total retrieval errors (Figure 9.b) account for most of the sharp variations observed between adjacent boxes. For instance, some of the retrievals located in $60^{\circ}$ - 

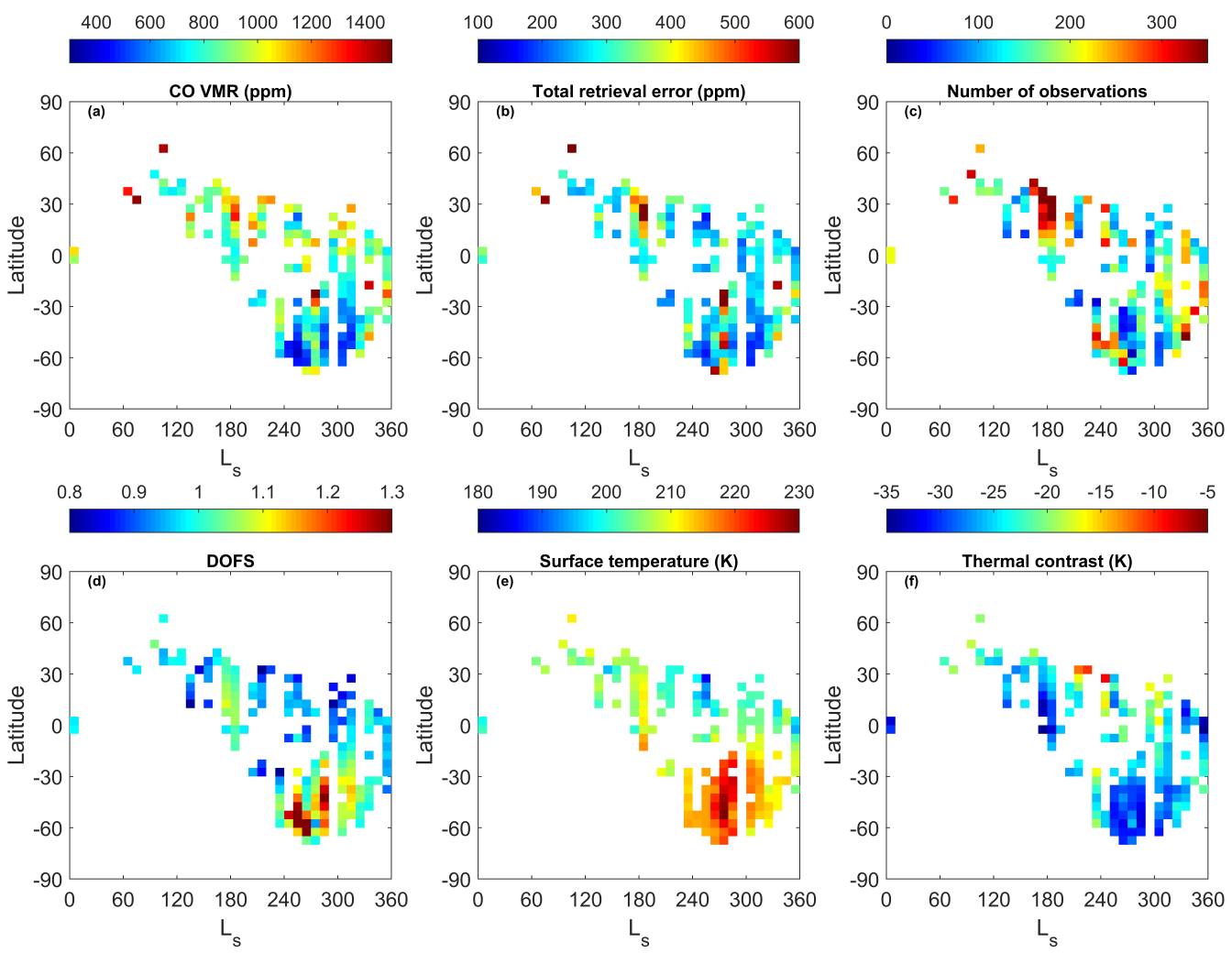

Figure 9: Seasonal cycle of the retrieved CO column-averaged VMR (ppm, a), of the associated total retrieval error (ppm, b), of the DOFS (d), of the surface temperature (K, e) and of the thermal contrast (K, f). Panel (c) shows the number of single PFS spectra averaged together.

$40^{\circ} \mathrm{S}$ and $250^{\circ}-300^{\circ} \mathrm{L}_{\mathrm{s}}$ has led to $\mathrm{CO}$ abundances unexpectedly high, of around 900-1000 ppm. These are associated to an error of 500-600 ppm, showing that the increase of the CO VMR is likely not real. The large CO VMRs $(1100-1400 \mathrm{ppm})$ observed in $20^{\circ}-40^{\circ} \mathrm{N}$ and in $150^{\circ}-200^{\circ} \mathrm{L}_{\mathrm{s}}$ are also associated with large errors (450-700 ppm), which could explain the sharp variations observed between the adjacent retrievals. Another, yet less important, reason for the variations observed is related to the fact that we analyse 
average spectra built from an inhomogeneous number of PFS observations (Figure 9.c), including different contributions of the different Martian Years (in terms of the number of spectra included in the average). Nevertheless, one significant feature of variability emerges from the uncertainty: it corresponds to the known decrease of the CO VMR in the Southern Hemisphere during spring and summer due to the sublimation of the $\mathrm{CO}_{2}$ ice. CO VMRs lower than $600 \mathrm{ppm}$ are indeed retrieved from $\mathrm{L}_{\mathrm{s}}=240^{\circ}$ southern to $-30^{\circ}$. Interestingly, these $\mathrm{CO}$ measurements are those associated with the largest DOFS as shown in Figure 9.d. The Southern Hemisphere in spring and summer is actually associated with the largest surface temperatures (larger than 210 K, Figure 9.e) and the highest negative thermal contrasts ( $<-25$ K, Figure 9.f). This is observed for the analysed set but also for Mars in general. These temperature conditions are the best night-time conditions to measure $\mathrm{CO}$ and allow therefore the best $\mathrm{CO}$ night-time retrievals in this region and at this period.

\section{Discussion and conclusion}

This work has demonstrated the possibility of retrieving the Martian CO abundance from night-time PFS nadir spectra in the SW channel, exploiting the fundamental 1-0 band of CO. Analysing such observations is challenging because the SNR of single spectra is very low. The detection of CO required first averaging a sufficiently large number of PFS night-time spectra. More specifically 8 MYs of PFS observations have been averaged together for boxes of $5^{\circ} \times 10^{\circ}$ (latitude $\times \mathrm{L}_{\mathrm{s}}$ ) to guarantee a sufficient SNR for CO. A total of 1163 averaged night-time spectra have been built, from which only a subset 
was kept based on a detection method using BTDs in $21 \mathrm{CO}$-sensitive spectral channels. A positive detection of the 1-0 band of $\mathrm{CO}$ was assumed when the mean BTDs, calculated over the 21 CO lines, is larger than the NEDT. 449 averaged spectra out of the 1163 turned out to have detectable CO lines and have been analysed using the Optimal Estimation method. CO vertical profiles have been successfully retrieved for 350 spectra and only 170 have been finally selected based on the quality of the fits.

These $170 \mathrm{CO}$ measurements are not randomly distributed, but are mostly located in the Southern Hemisphere during spring and summer. Actually, one of the main result revealed in this work is that night-time $\mathrm{CO}$ is the most favourably measured from PFS observations in this region at this period. The well-known decrease of the CO VMR, related to the sublimation of $\mathrm{CO}_{2}$ at the South Pole, is indeed well observed in the night-time seasonal cycle measured in this work, thanks to the combination of two favourable conditions: high surface temperatures (larger than $210 \mathrm{~K}$ ) and high negative thermal contrasts $(<-20 \mathrm{~K})$. High surface temperatures contribute to increase the SNR of single spectra while high thermal contrasts (negative or positive) favour the sensitivity to $\mathrm{CO}$ in the near-surface atmosphere. In the Southern Hemisphere in spring and summer, these two conditions are such that it is actually possible to measure the $\mathrm{CO}$ abundance from average spectra built using a smaller number of PFS observations, for instance recorded in the same MY. Figure 10 shows an example of such spectrum. It has been built using only 24 single night-time PFS observations recorded in MY 29 at $238^{\circ}<\mathrm{L}_{\mathrm{s}}<253^{\circ}$ and located in a box of $10^{\circ} \times 4^{\circ}$ (longitude $\times$ latitude) If this kind of average is possible for all MY, it would allow studying the 
inter annual variability of night-time CO in the Southern Hemisphere during spring and summer; this could be investigated by future works. Note also that relaxing the criterion on dust and ice optical depths, could allow taking into account more spectra and improve further the results. Finally, given the two conditions required to detect $\mathrm{CO}$ during night with PFS, we anticipate that it will remain difficult to measure the $\mathrm{CO}$ abundance during the polar night, as the surface temperatures are extremely low (around 140-150 K).
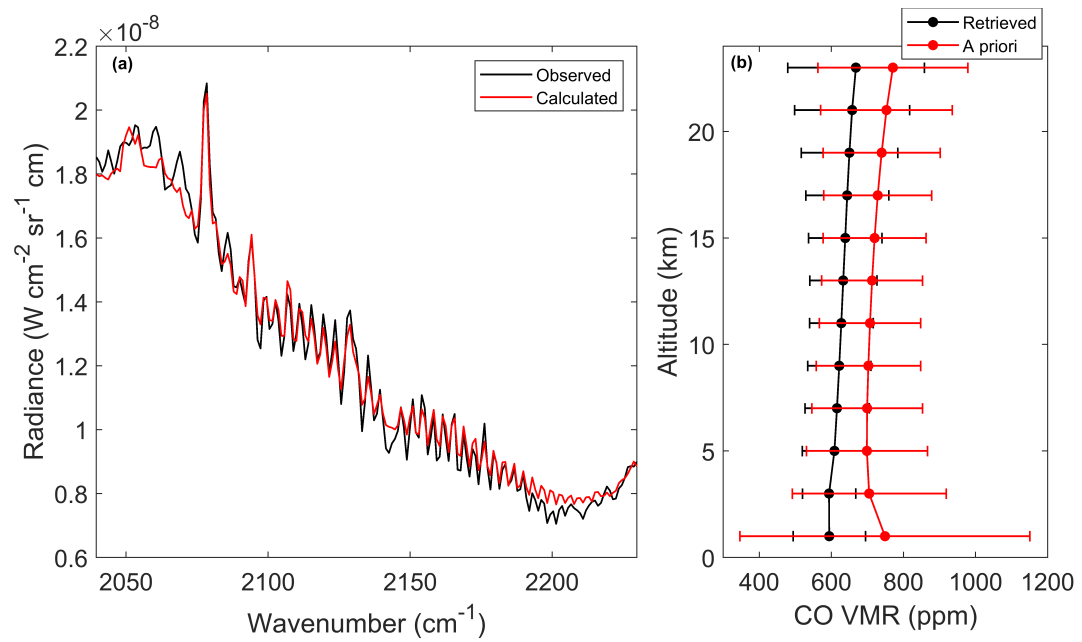

Figure 10: (a) Example of average night-time PFS spectrum built using only spectra recorded in the same MY (see details in the text). A test retrieval has been performed and the result is in red. (b) CO vertical profile retrieved from the spectrum shown in (a). the thermal inversions, night-time PFS observations provide stronger con- 
straints on the near-surface $\mathrm{CO}$ abundance than daytime observations, which carry information mostly for the $0-20 \mathrm{~km}$ atmospheric region. However, it is important to remind that, because of their low SNR, night-time PFS observations provide $\mathrm{CO}$ measurements with lower spatial and temporal resolutions than daytime observations. In addition to the vertical sensitivity, a complete error budget, including the propagation of two types of temperature uncertainties, has been performed. We have shown that the retrieval error on the 0-24 km CO column is within 6-15\% for a large majority of the retrievals and depends on the amount of $\mathrm{CO}$ in the atmosphere. This error is similar to that characterizing daytime CO measurements from single PFS spectra. In addition to this, we have estimated that an extra $6 \%$ of error comes from the temperature retrieval error and another $6 \%$ comes from the temperature uncertainty due to our hypothesis on the surface pressure and pressure profile. Note that the contribution of the uncertainty on the surface pressure to the error on the $\mathrm{CO}$ abundance is included in the temperature pressure error. A last source of uncertainty comes from the ILS formulation and has been estimated to be around 14\%. Assuming that these errors linearly add up, the total error on the CO 0-24 km column is within $32-41 \%$ on average. Finally, we have shown that the DOFS values are larger than 0.9 for the majority of the night-time retrievals, and this is also slightly larger than what has been observed for daytime PFS observations.

In conclusion, retrieving the $\mathrm{CO}$ abundance from night-time PFS observations is a difficult task requiring spectral averaging and careful fitting and analysis. However, this exploratory work has firmly demonstrated that it was possible in favourable periods and regions. For these, the night-time 
$\mathrm{CO}$ measurements contribute to significantly constrain the near-surface CO abundance and they are therefore expected to be an important complement of the highly resolved profiles retrieved from solar occultation and limb measurements.

\section{Acknowledgements}

This work is partly funded by the F.R.S. FNRS CRAMIC project under grant number T.0171.16. The PFS experiment was built at the Institute for Space Astrophysics and Planetology (formerly the Institute for Interplanetary Space Physics) of the National Institute for Astrophysics, and is currently funded by the Italian Space Agency (agreement number 2018-2HH.0) in the context of the science activities for NOMAD-ExoMars and for PFS-MEx. Sophie Bauduin is "Chargée de recherches"' with F.R.S.-FNRS. The authors would like to thank the LMD for providing the access to the Mars Climate Database, which was used to build the a priori vertical profile $\mathrm{CO}$ and the associated covariance matrix.

\section{References}

Atreya, S.K., Gu, Z.G., 1994. Stability of the Martian atmosphere: Is heterogeneous catalysis essential? J. Geophys. Res. 99, 13133-13145. doi:10.1029/94JE01085.

Atreya, S.K., Gu, Z.G., 1995. Photochemistry and stability of the atmosphere of Mars. Adv. Space Res. 16. doi:10.1016/0273-1177(95)00250-I.

Barret, B., Hurtmans, D., Carleer, M.R., De Mazière, M., Mahieu, E., Coheur, P.F., 2005. Line narrowing effect on the retrieval of $\mathrm{HF}$ and $\mathrm{HCl}$ 
vertical profiles from ground-based FTIR measurements. J. Quant. Spectrosc. Radiat. Transfer 95, 499-519. doi:10.1016/j.jqsrt.2004.12.005.

Bauduin, S., Clarisse, L., Theunissen, M., George, M., Hurtmans, D., Clerbaux, C., Coheur, P.F., 2017. IASI's sensitivity to near-surface carbon monoxide (CO): Theoretical analyses and retrievals on test cases. Journal of Quantitative Spectroscopy and Radiative Transfer 189, 428-440. doi:10.1016/j.jqsrt.2016.12.022.

Bauduin, S., Giuranna, M., Wolkenber, P., Nardi, L., Daerden, F., Bouche, J., Wespes, C., Lecomte, G., Vandaele, A.C., Coheur, P., 2020. Exploiting night-time averaged spectra from PFS/MEX shortwave channel. Part 1: Temperature retrieval from the $\mathrm{CO}_{2} \nu_{3}$ band. Planetary and Space Science (submitted) .

Billebaud, F., Brillet, J., Lellouch, E., Fouchet, T., Encrenaz, T., Cottini, V., Ignatiev, N., Formisano, V., Giuranna, M., Maturilli, A., Forget, F., 2009. Observations of $\mathrm{CO}$ in the atmosphere of Mars with PFS onboard Mars Express. Planet. Space Sci. 57, 1446-1457. doi:10.1016/j .pss .2009.07. 004.

Bouche, J., Bauduin, S., Giuranna, M., Robert, S., Aoki, S., Vandaele, A.C., Erwin, J.T., Daerden, F., Wolkenberg, P., Coheur, P.F., 2019. Retrieval and characterization of carbon monoxide $(\mathrm{CO})$ vertical profiles in the Martian atmosphere from observations of PFS/MEX. J. Quant. Spectrosc. Radiat. Transfer doi:10.1016/j.jqsrt.2019.05.009.

Bouche, J., Coheur, P.F., Giuranna, M., Wolkenberg, P., Nardi, L., Amoroso, 
M., Vandaele, A.C., Daerden, F., Neary, L., Bauduin, S., 2020. Seasonal and spatial variability of carbon monoxide $(\mathrm{CO})$ in the Martian atmosphere from PFS/MEX observations (submitted to JGR Planets).

Clancy, R.T., Muhleman, D.O., Jakosky, B.M., 1983. Variability of carbon monoxide in the Mars atmosphere. Icarus 55, 282-301.

Coheur, P.F., Barret, B., Turquety, S., Hurtmans, D., Hadji-Lazaro, J., Clerbaux, C., 2005. Retrieval and characterization of ozone vertical profiles from a thermal infrared nadir sounder. J. Geophys. Res. 110, D24303. doi:10.1029/2005JD005845.

Daerden, F., Neary, L., Viscardy, S., García Muñoz, A., Clancy, R., Smith, M., Encrenaz, T., Fedorova, A., 2019. Mars atmospheric chemistry simulations with the GEM-Mars general circulation model. Icarus 326, 197 224. doi:https://doi.org/10.1016/j.icarus.2019.02.030.

Encrenaz, T., Fouchet, T., Melchiorri, R., Drossart, P., Gondet, B., Langevin, Y., Bibring, J.P., Forget, F., Bézard, B., 2006. Seasonal variations of the martian CO over Hellas as observed by OMEGA/Mars Express. Astron. Astrophys. 459, 265-270. doi:10.1051/0004-6361:20065586.

Erwin, J.T., Aoki, S., Thomas, I.R., Trompet, L., Vandaele, A.C., Robert, S., Daerden, F., Ristic, B., Villanueva, G.L., Liuzzi, G., Lopez-Moreno, J.J., Bellucci, G., Patel, M.R., 2019. Martian Atmosphere CO Vertical Profiles: Results from the First Year of TGO/NOMAD Science Operations, in: EPSC-DPS Joint Meeting 2019, pp. EPSC-DPS2019-1471-1. 
Forget, F., Hourdin, F., Fournier, R., Hourdin, C., Talagrand, O., Collins, M., Lewis, S.R., Read, P.L., Huot, J.P., 1999. Improved general circulation models of the Martian atmosphere from the surface to above $80 \mathrm{~km}$. J. Geophys. Res. 104, 24155-24176. doi:10.1029/1999JE001025.

Forget, F., Millour, E., Montabone, L., Lefèvre, F., 2008. Non Condensable Gas Enrichment and Depletion in the Martian Polar Regions, in: Third International Workshop on The Mars Atmosphere: Modeling and Observations, p. 9106.

Formisano, V., Angrilli, F., Arnold, G., Atreya, S., Bianchini, G., Biondi, D., Blanco, A., Blecka, M.I., Coradini, A., Colangeli, L., Ekonomov, A., Esposito, F., Fonti, S., Giuranna, M., Grassi, D., Gnedykh, V., Grigoriev, A., Hansen, G., Hirsh, H., Khatuntsev, I., Kiselev, A., Ignatiev, N., Jurewicz, A., Lellouch, E., Lopez Moreno, J., Marten, A., Mattana, A., Maturilli, A., Mencarelli, E., Michalska, M., Moroz, V., Moshkin, B., Nespoli, F., Nikolsky, Y., Orfei, R., Orleanski, P., Orofino, V., Palomba, E., Patsaev, D., Piccioni, G., Rataj, M., Rodrigo, R., Rodriguez, J., Rossi, M., Saggin, B., Titov, D., Zasova, L., 2005. The Planetary Fourier Spectrometer (PFS) onboard the European Mars Express mission. Planet. Space Sci. 53, 963-974. doi:10.1016/j.pss.2004.12.006.

Giuranna, M., Formisano, V., Biondi, D., Ekonomov, A., Fonti, S., Grassi, D., Hirsch, H., Khatuntsev, I., Ignatiev, N., Malgoska, M., Mattana, A., Maturilli, A., Mencarelli, E., Nespoli, F., Orfei, R., Orleanski, P., Piccioni, G., Rataj, M., Saggin, B., Zasova, L., 2005a. Calibration of the Planetary 
Fourier Spectrometer long wavelength channel. Planet. Space Sci. 53, 9931007. doi:10.1016/j.pss.2005.02.007.

Giuranna, M., Formisano, V., Biondi, D., Ekonomov, A., Fonti, S., Grassi, D., Hirsch, H., Khatuntsev, I., Ignatiev, N., Michalska, M., Mattana, A., Maturilli, A., Moshkin, B.E., Mencarelli, E., Nespoli, F., Orfei, R., Orleanski, P., Piccioni, G., Rataj, M., Saggin, B., Zasova, L., 2005b. Calibration of the Planetary Fourier Spectrometer short wavelength channel. Planet. Space Sci. 53, 975-991. doi:10.1016/j.pss.2004.12.007.

Giuranna, M., Viscardy, S., Daerden, F., Neary, L., Etiope, G., Oehler, D., Formisano, V., Aronica, A., Wolkenberg, P., Aoki, S., CardesínMoinelo, A., Marín-Yaseli de la Parra, J., Merritt, D., Amoroso, M., 2019a. Independent confirmation of a methane spike on Mars and a source region east of Gale Crater. Nature Geoscience 12, 326-332. doi:10.1038/s41561-019-0331-9.

Giuranna, M., Wolkenberg, P., Grassi, D., Aronica, A., Aoki, S., Scaccabarozzi, D., Saggin, B., Formisano, V., 2019b. The current weather and climate of Mars: 12 years of atmospheric monitoring by the Planetary Fourier Spectrometer on Mars Express. Icarus doi:10.1016/j.icarus. 2019.113406.

Gordon, I.E., Rothman, L.S., Hill, C., Kochanov, R.V., Tan, Y., Bernath, P.F., Birk, M., Boudon, V., Campargue, A., Chance, K.V., Drouin, B.J., Flaud, J.M., Gamache, R.R., Hodges, J.T., Jacquemart, D., Perevalov, V.I., Perrin, A., Shine, K.P., Smith, M.A.H., Tennyson, J., Toon, G.C., 
Tran, H., Tyuterev, V.G., Barbe, A., Császár, A.G., Devi, V.M., Furtenbacher, T., Harrison, J.J., Hartmann, J.M., Jolly, A., Johnson, T.J., Karman, T., Kleiner, I., Kyuberis, A.A., Loos, J., Lyulin, O.M., Massie, S.T., Mikhailenko, S.N., Moazzen-Ahmadi, N., Müller, H.S.P., Naumenko, O.V., Nikitin, A.V., Polyansky, O.L., Rey, M., Rotger, M., Sharpe, S.W., Sung, K., Starikova, E., Tashkun, S.A., Auwera, J.V., Wagner, G., Wilzewski, J., Wcisło, P., Yu, S., Zak, E.J., 2017. The HITRAN2016 molecular spectroscopic database. Journal of Quantitative Spectroscopy and Radiative Transfer 203, 3-69. doi:10.1016/j.jqsrt.2017.06.038.

Holmes, J.A., Lewis, S.R., Patel, M.R., Smith, M.D., 2019. Global analysis and forecasts of carbon monoxide on Mars. Icarus 328, 232-245.

Kaplan, L.D., Connes, J., Connes, P., 1969. Carbon Monoxide in the Martian Atmosphere. The Astrophysical Journal 157, L187. doi:10.1086/180416.

Korablev, O., Montmessin, F., Trokhimovskiy, A., Fedorova, A.A., Shakun, A.V., Grigoriev, A.V., Moshkin, B.E., Ignatiev, N.I., Forget, F., Lefèvre, F., Anufreychik, K., Dzuban, I., Ivanov, Y.S., Kalinnikov, Y.K., Kozlova, T.O., Kungurov, A., Makarov, V., Martynovich, F., Maslov, I., Merzlyakov, D., Moiseev, P.P., Nikolskiy, Y., Patrakeev, A., Patsaev, D., Santos-Skripko, A., Sazonov, O., Semena, N., Semenov, A., Shashkin, V., Sidorov, A., Stepanov, A.V., Stupin, I., Timonin, D., Titov, A.Y., Viktorov, A., Zharkov, A., Altieri, F., Arnold, G., Belyaev, D.A., Bertaux, J.L., Betsis, D.S., Duxbury, N., Encrenaz, T., Fouchet, T., Gérard, J.C., Grassi, D., Guerlet, S., Hartogh, P., Kasaba, Y., Khatuntsev, I., Krasnopolsky, V.A., Kuzmin, R.O., Lellouch, E., Lopez-Valverde, M.A., 
Luginin, M., Määttänen, A., Marcq, E., Martin Torres, J., Medvedev, A.S., Millour, E., Olsen, K.S., Patel, M.R., Quantin-Nataf, C., Rodin, A.V., Shematovich, V.I., Thomas, I., Thomas, N., Vazquez, L., Vincendon, M., Wilquet, V., Wilson, C.F., Zasova, L.V., Zelenyi, L.M., Zorzano, M.P., 2018. The Atmospheric Chemistry Suite (ACS) of Three Spectrometers for the ExoMars 2016 Trace Gas Orbiter. Space Sci. Rev. 214, 7. doi:10.1007/s11214-017-0437-6.

Krasnopolsky, V.A., 2003. Spectroscopic mapping of Mars CO mixing ratio: Detection of north-south asymmetry. Journal of Geophysical Research (Planets) 108, 5010. doi:10.1029/2002JE001926.

Krasnopolsky, V.A., 2015. Variations of carbon monoxide in the martian lower atmosphere. Icarus 253, 149-155. doi:10.1016/j.icarus.2015.03. 006.

McElroy, M.B., Donahue, T.M., 1972. Stability of the Martian Atmosphere. Technical Report.

Olsen, K.S., Alday, J., Baggio, L., Belyaev, D., Fedorova, A., Grigoriev, A., Lefèvre, F., Korablev, O., Montmessin, F., Patrakeev, A., Rossi, L., Shakun, A., Trokhimovskiy, A., 2019. Vertical distributions of $\mathrm{CO}, \mathrm{H}_{2} \mathrm{O}$, HDO, and temperature in the atmosphere of Mars after a year of observations with the ExoMars Trace Gas Orbiter (TGO) Atmospheric Chemistry Suite mid-infrared (ACS MIR) solar occultation spectrometer, in: EPSCDPS Joint Meeting 2019, pp. EPSC-DPS2019-1463-3.

Parkinson, T.D., Hunten, D.M., 1972. Spectroscopy and Acronomy of $\mathrm{O}_{2}$ 
on Mars. Journal of Atmospheric Sciences 29, 1380-1390. doi:10.1175/ $1520-0469$ (1972) 029<1380:SAA000>2 . 0. CO ; 2.

Poulet, F., Quantin-Nataf, C., Ballans, H., Dassas, K., Audouard, J., Carter, J., Gondet, B., Lozac'h, L., Malapert, J.C., Marmo, C., Riu, L., Séjourné, A., 2018. PSUP: A Planetary SUrface Portal. Planet. Space Sci. 150, 2-8. doi:10.1016/j.pss. 2017.01.016.

Quantin-Nataf, C., Lozac'h, L., Thollot, P., Loizeau, D., Bultel, B., Fernando, J., Allemand, P., Dubuffet, F., Poulet, F., Ody, A., Clenet, H., Leyrat, C., Harrisson, S., 2018. MarsSI: Martian surface data processing information system. Planetary and Space Science 150, 157-170. doi:10.1016/j.pss. 2017.09.014.

Rodgers, C.D., 2000. Inverse Methods for Atmospheric Sounding: Theory and Practice. World scientific.

Sindoni, G., Formisano, V., Geminale, A., 2011. Observations of water vapour and carbon monoxide in the Martian atmosphere with the SWC of PFS/MEX. Planet. Space Sci. 59, 149-162. doi:10.1016/j.pss . 2010. 12.006 .

Smith, M.D., Daerden, F., Neary, L., Khayat, A., 2018. The climatology of carbon monoxide and water vapor on Mars as observed by CRISM and modeled by the GEM-Mars general circulation model. Icarus 301, 117-131. doi:10.1016/j . icarus. 2017.09.027.

Vandaele, A.C., Lopez-Moreno, J.J., Patel, M.R., Bellucci, G., Daerden, F., Ristic, B., Robert, S., Thomas, I.R., Wilquet, V., Allen, M., Alonso- 
Rodrigo, G., Altieri, F., Aoki, S., Bolsée, D., Clancy, T., Cloutis, E., Depiesse, C., Drummond, R., Fedorova, A., Formisano, V., Funke, B., González-Galindo, F., Geminale, A., Gérard, J.C., Giuranna, M., Hetey, L., Ignatiev, N., Kaminski, J., Karatekin, O., Kasaba, Y., Leese, M., Lefèvre, F., Lewis, S.R., López-Puertas, M., López-Valverde, M., Mahieux, A., Mason, J., McConnell, J., Mumma, M., Neary, L., Neefs, E., Renotte, E., Rodriguez-Gomez, J., Sindoni, G., Smith, M., Stiepen, A., Trokhimovsky, A., Vander Auwera, J., Villanueva, G., Viscardy, S., Whiteway, J., Willame, Y., Wolff, M., 2018. NOMAD, an Integrated Suite of Three Spectrometers for the ExoMars Trace Gas Mission: Technical Description, Science Objectives and Expected Performance. Space Sci. Rev. 214, 80. doi:10.1007/s11214-018-0517-2.

Wolkenberg, P., Giuranna, M., Grassi, D., Aronica, A., Aoki, S., Scaccabarozzi, D., Saggin, B., 2018. Characterization of dust activity on Mars from MY27 to MY32 by PFS-MEX observations. Icarus 310, 3247. doi:10.1016/j . icarus. 2017.10 .045 . 